\title{
The Impact of an Antenna Array in a Relay Network
}

\author{
Ramachandran Rajagopalan, Daryl Reynolds, Matthew C. Valenti, and Brian D. Woerner \\ Lane Department of Computer Science and Electrical Engineering \\ West Virginia University, Morgantown, West Virginia 26505-0250 \\ Email: chandran.rc@gmail.com,\{Daryl.Reynolds, Matthew.Valenti,Brian.Woerner\}@mail.wvu.edu
}

\begin{abstract}
Cooperative diversity is a form of distributed spacetime macrodiversity capable of mitigating the detrimental effects of multipath fading in a wireless network. Prior work on cooperative diversity has focused on the case that all terminals have a single antenna. However, in many practical situations, it is feasible for one or more terminals to be equipped with an array of multiple antennas. This paper investigates the impact of the presence of a single antenna array in a three terminal orthogonal relaying network. The array may be at the source, relay, or destination. The information outage probability under diversity combining is derived, and closed form expressions given wherever possible. The numerical results suggest that it is best to place the array at the destination, and that it is better to place the array at the relay than at the source.
\end{abstract}

Keywords: Cooperative diversity, decode and forward, relay channel, Multiple antennas, Outage probability.

\section{INTRODUCTION}

Multipath fading is one of the most challenging phenomena in wireless communications. Multipath is commonly mitigated through the use of diversity techniques, including time-, frequency-, spatial-, and antenna-diversity (with the latter two often used interchangeably). In [1], [2], the impact of the above mentioned diversity schemes are studied extensively in a pointpoint communications scenario. Because diversity is generally applied in a nonergodic setting, where the Shannon capacity does not exist, the benefits of diversity are explained using the concepts of information outage probability or outage capacity. Recent research [3], [4] indicates a special form of spatial diversity called cooperative diversity is an effective alternative for mitigating fading in wireless networks.

Cooperative diversity is achieved in a relay network, wherein a relay assists the source transmit a message to the destination. Often, the relay operates in a time-division duplex (TDD) mode, which greatly simplifies system implementation. In addition, the source and relay usually transmit orthogonal signals. Orthogonality could be achieved by using different time slots [4], orthogonal spreading codes[3], [?], or an orthogonal space-time code [5]. While it is possible to engineer systems that do not require orthogonal source and relay transmissions, for ease of exposition we assume that this condition is met for the remainder of this discussion.

Two fundamental methods of orthogonal relaying considered in the literature are amplify-and-forward (AF) and decode-and-forward (DF) relaying [4]. In AF relaying, the relay simply retransmits an amplified version of the received signal, without making hard decisions on the message. The main drawback of the AF relaying protocol is that the noise on the source-relay channel is present in the retransmitted signal. In conventional (nonadaptive) DF relaying, the relay decodes and retransmits a reconstructed version of the received signal. The main drawback in this relaying scheme is that the relay could retransmit an incorrectly decoded message, though this problem can be alleviated through the use of an error detecting code. Coded cooperation is an efficient variation on the idea of DF relaying [6]. Whereas a standard DF protocol might have the source and relay transmit the same codeword (through repetition coding), a coded cooperation protocol would have the source and relay transmit different parts of the same codeword (through incremental redundancy).

Prior work on cooperative diversity has generally considered networks with only a single antenna at each node, while the use of antenna arrays at the individual nodes has gone largely unnoticed. In [5], the source and relay nodes form a distributed antenna array and are used to send a space-time codeword. However, each node contains only a single antenna. In [7], the base station (source) uses two antennas to send the 2by-2 Alamouti space-time code to a pair of handsets (source and destination). Each handset is equipped with one antenna, but are able to mimic a two antenna receiver by exchanging information. While this is an example of a relay network with an array, only one configuration was considered and a more generalized study should include the possibility of placing the array at a different terminal or using arrays of more than just two antennas. In [8], a unifying analysis is presented for the case of AF protocols with multiple antenna terminals, but DF was not considered. In [9], DF protocols with multiple antenna terminals was considered, but explicit, closed form expressions for outage probability were not given (instead numerical results relied on Monte Carlo integration). In [6], code combining techniques for networks with single antenna terminals is considered in depth. [10] provides a unified analysis of AF and DF protocols and proposes new adaptive relaying protocols using code and diversity combining techniques. While the adaptive cooperative diversity for the multiple relay case is considered to be a generalization of hybrid-ARQ, again only terminals with a single antenna are considered.

In this paper, we consider the impact of the presence of an antenna array in a relay network with a single relay. 
The antenna array may be placed at the source, relay, or destination. Our focus is on the adaptive DF protocol with diversity combining, whereby the relay only retransmits if it successfully decodes the source's message (under the assumption of a perfect error detection code). An informationtheoretic approach is taken, and our main performance metric is information outage probability. By limiting the array to be at only one of the three nodes, we are able to derive exact expressions for the information outage probabilities. The key contribution of the paper is equations (16), (20), and (24), which give the information outage probability when the array is at the destination, relay, and source, respectively. For the case that the array is at the destination, a closed form expression is obtained, while for the cases that the array is at the relay or source, the expression contains a one-dimensional integral which can be solved numerically. Numerical results illustrate the potential benefits of the use of the array.

\section{Point-to-Point MiMo Channels}

The discrete-time matrix-vector received signal model for a multiple-input multiple-output (MIMO) system with $M_{T}$ transmit antennas and $M_{R}$ receive antennas is

$$
\mathbf{y}=\mathbf{H s}+\mathbf{n}
$$

where

$$
\mathbf{y}=\left[\begin{array}{lllll}
y_{1} & y_{2} & \cdot & \cdot & y_{M_{R}}
\end{array}\right]^{T}
$$

is a vector of matched filter outputs, one for each receive antenna ( $T$ indicates vector transpose), and

$$
\mathbf{H}=\left[\begin{array}{cccc}
h_{1,1} & h_{1,2} & \cdots & h_{1, M_{T}} \\
\vdots & \vdots & & \vdots \\
h_{M_{R}, 1} & h_{M_{R}, 2} & \cdots & h_{M_{R}, M_{T}}
\end{array}\right]
$$

is the channel gain matrix, where $h_{i, j}$ is the complex channel gain between transmit antenna $j$ and receive antenna $i$. The channel is considered to be spatially white, so that each channel gain is i.i.d. complex Gaussian with unit power. Also,

$$
\mathbf{s}=\left[\begin{array}{lllll}
s_{1} & s_{2} & \cdot & \cdot & s_{M_{T}}
\end{array}\right]^{T}
$$

is a vector of transmitted symbols, one from each transmit antenna, and $\mathbf{n}$ is a length $M_{R}$ column vector of i.i.d white noise, where each element is Gaussian with variance $N_{o}$.

The instantaneous capacity of the MIMO channel with channel state information at the receiver is given in [1] as

$$
C=\log \operatorname{det}\left[\mathbf{I}_{M_{R}}+\frac{\rho}{M_{T}} \mathbf{H H}^{\dagger}\right],
$$

where $\rho=P / \sigma^{2}$ is the ratio of transmitted signal power $(P)$ to noise power $\left(\sigma^{2}\right)$ over a unit gain (unfaded) channel, $\mathbf{I}_{n}$ is the n-by-n identity matrix, and $\mathbf{H}^{\dagger}$ is the Hermetian transpose of $\mathbf{H}$. All logarithms in this paper are taken to the base-2 so that capacity takes on units of bits per channel use.

We are concerned with two types of point-to-point links, the single-input, multiple-output (SIMO) link (for which $M_{T}=1$ ) and the multiple-input, single-output (MISO) link $\left(M_{R}=1\right)$.

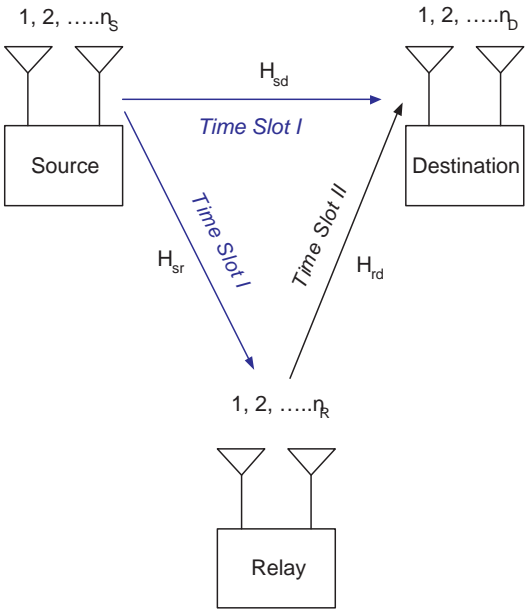

Fig. 1. A three terminal relay network.

For these cases, the matrix $\mathbf{H}$ becomes either a row (MISO case) or column (SIMO case) vector $\mathbf{h}$ and the capacity expression reduces to

$$
C=\log \left(1+\frac{\rho}{M_{T}}\|\mathbf{h}\|^{2}\right),
$$

where $\|\mathbf{h}\|^{2}=\sum_{i}\left|h_{i}\right|^{2}$

For transmission with target rate $R$ [bps/Hz], the channel is said to be in an outage if $C<R$. For the SIMO and MISO channels, the information outage probability is:

$$
\begin{aligned}
\operatorname{Pr}(C<R) & =\operatorname{Pr}\left[\log \left(1+\frac{\rho}{M_{T}}\|\mathbf{h}\|^{2}\right)<R\right] \\
& =\operatorname{Pr}[\underbrace{\|\mathbf{h}\|^{2}}_{Y}<M_{T} \underbrace{\left(\frac{\left(2^{R}-1\right)}{\rho}\right)}_{z_{1}}] \\
& =1-e^{-M_{T} z_{1}} \sum_{k=0}^{L-1} \frac{\left(M_{T} z_{1}\right)^{k}}{k !}
\end{aligned}
$$

where $L=\max \left(M_{T}, M_{R}\right)$. In (7), $Y$ follows a chi squared $\left(\chi^{2}\right)$ distribution with $n=2 L$ degrees of freedom (DOF), and therefore its CDF may be obtained in closed form (see, for instance [11]).

\section{RELAY NETWORKS}

Consider the three terminal network shown in Fig. 1. Let $n_{S}, n_{R}$, and $n_{D}$ denote the number of antennas at the source, relay, and destination, respectively. The source and relay transmit with identical power, and if a particular terminal uses multiple transmit antennas, the power is divided across that terminal's antennas. To remove the effects of topology, we assume that the path loss between terminals is identical, which is true if the three terminals are located at the corners of an equilateral triangle. The channels between nodes are independent, and each is a quasi-static Rayleigh flat fading channel. Communication takes place through two time slots of equal duration. In the first slot, the source transmits and both relay and destination listen. In the second slot, the relay will 
retransmit the initial source message if the relay successfully decoded the source's initial transmission (c.f. adaptive DF).

The signal at the relay due to the source transmission is

$$
\mathbf{y}_{s r}=\mathbf{H}_{s r} \mathbf{s}_{s}+\mathbf{n}_{r}
$$

while the source's signal at the destination is

$$
\mathbf{y}_{s d}=\mathbf{H}_{s d} \mathbf{s}_{s}+\mathbf{n}_{d_{1}}
$$

where $\mathbf{H}_{s r}$ is the $n_{R} \times n_{S}$ channel gain matrix for the sourcerelay channel, $\mathbf{H}_{s d}$ is the $n_{D} \times n_{S}$ channel gain matrix for the source-destination channel, $\mathbf{s}_{s}$ is a length $n_{S} \times 1$ transmitted signal vector, and $\mathbf{n}_{r}, \mathbf{n}_{d_{1}}$ are the respective channel noise vectors drawn from an ensemble of i.i.d complex Gaussian random variables with zero mean and variance $\sigma^{2}=N_{o}$.

If the relay decodes correctly, it retransmits the message to the destination during the second slot. The signal at the destination due to the relay transmission is

$$
\mathbf{y}_{r d}=\mathbf{H}_{r d} \mathbf{s}_{r}+\mathbf{n}_{d_{2}}
$$

where $\mathbf{H}_{r d}$ is the $n_{D} \times n_{R}$ channel gain matrix of the of the relay-destination path, $\mathbf{s}_{r}$ is a length $n_{R} \times 1$ transmitted signal vector, and $\mathbf{n}_{d_{2}}$ is a vector of i.i.d. Gaussian noise vector with zero mean and variance $\sigma^{2}=N_{o}$.

When $n_{R}=n_{S}$ and the relay decodes the message successfully, $\mathbf{s}_{r}=\mathbf{s}_{s}$ and the destination maximal ratio (MRC) combines the received source and relay signals in the usual way. However, if $n_{R} \neq n_{S}$, then $\mathbf{s}_{r}$ cannot equal $\mathbf{s}_{s}$, since the vectors are of differing lengths. In this case, we may assume a two stage encoding process, whereby the message is first encoded with a channel code (defined over an arbitrarily large alphabet) and then modulated using a vector modulator (for instance a space-time code). Both source and relay transmit the same channel codeword, though the modulated symbol vectors will necessarily be different. At the destination, the source and relay signals are individually demodulated, and the demodulator transforms the channel symbol likelihoods into code symbol likelihoods. Since both source and relay transmit the same underlying channel code, the code symbol likelihoods corresponding to the source transmission may be MRC combined with the code symbol likelihoods corresponding to the relay transmission, and the aggregate codeword can be passed through the channel decoder.

\section{Relay Network Outage Analysis}

The instantaneous capacity of adaptive DF relaying is [12]

$$
C_{D F}=\max \left\{\min \left\{\frac{1}{2} C_{(s d, r d)}, \frac{1}{2} C_{s r}\right\}, \frac{1}{2} C_{s d}\right\}
$$

where $C_{s d}$ is the instantaneous capacity of the sourcedestination (S-D) path, $C_{s r}$ is the instantaneous capacity of source-relay (S-R) path, and $C_{(s d, r d)}$ is the instantaneous capacity at destination from the combined signal S-D and relay-destination (R-D) paths. An outage occurs when the instantaneous capacity is less than the transmission rate $R$ i.e.
$C_{D F}<R$. The corresponding information outage probability is

$$
\begin{aligned}
\mathbf{p}^{\text {outage }} & =\operatorname{Pr}\left(C_{D F}<R\right) \\
& =\operatorname{Pr}\left(C_{s r}<2 R\right) \operatorname{Pr}\left(C_{s d}<2 R\right) \\
& +\operatorname{Pr}\left(C_{s r}>2 R\right) \operatorname{Pr}\left(C_{(s d, r d)}<2 R\right)
\end{aligned}
$$

We now evaluate this expression for our three cases of interest, i.e. the array located at the destination, relay, and source.

\section{A. Array at the Destination}

When $n_{S}=1, n_{R}=1$, and $n_{D}=L, \mathbf{H}_{s d}=\mathbf{h}_{s d}$ and $\mathbf{H}_{r d}=\mathbf{h}_{r d}$ are length $\mathrm{L}$ column vectors, since the S-D and R-D channels are both SIMO, and $\mathbf{H}_{s r}=h_{s r}$ is scalar since the S-R channel is single-input, single-output (SISO). The computation of information outage probability $\operatorname{Pr}\left(C_{s d}<2 R\right)$ is identical to that of the direct transmission case given by (7) except that to account for the factor of 2 , we must replace $z_{1}$ with $z=\left(2^{2 R}-1\right) / \rho$. Making this substitution yields

$$
\operatorname{Pr}\left(C_{s d}<2 R\right)=1-e^{-z} \sum_{k=0}^{L-1} \frac{z^{k}}{k !} .
$$

Considering the SISO S-R link, we get

$$
\begin{aligned}
\operatorname{Pr}\left(C_{s r}<2 R\right) & =\operatorname{Pr}\left[\log \left(1+\rho\left|h_{s r}\right|^{2}\right)<2 R\right] \\
& =\operatorname{Pr}[\left|h_{s r}\right|^{2}<\underbrace{\left.\frac{2^{2 R}-1}{\rho}\right]}_{z} \\
& =1-e^{-z},
\end{aligned}
$$

since $\left|h_{s r}\right|^{2}$ is exponential.

The term $\operatorname{Pr}\left(C_{(s d, r d)}<2 R\right)$ represents the information outage probability due to the diversity combining at destination from the two SIMO paths S-D and R-D. The information outage of the diversity combined paths is

$$
\begin{aligned}
& \operatorname{Pr}\left(C_{(s d, r d)}<2 R\right)=\operatorname{Pr}\left[\log \left(1+\rho\left(\left\|\mathbf{h}_{s d}\right\|^{2}+\left\|\mathbf{h}_{r d}\right\|^{2}\right)\right)<2 R\right] \\
&=\operatorname{Pr}[(\underbrace{\left\|\mathbf{h}_{s d}\right\|^{2}}_{X}+\underbrace{\left\|\mathbf{h}_{r d}\right\|^{2}}_{Y})<\underbrace{\left.\frac{2^{2 R}-1}{\rho}\right]}_{z} \\
&=1-e^{-z} \sum_{k=0}^{2 L-1} \frac{z^{k}}{k !} .
\end{aligned}
$$

Here $X, Y$ are independent $\chi^{2}$ random variables, each with $2 L$ DOF, and therefore $X+Y$ is $\chi^{2}$ with $4 L$ DOF. The expression (15) is merely the CDF of $X+Y$ [11].

Substituting (13), (14), and (15) into (12), we arrive at the following closed form expression for information outage probability for this case (i.e. $n_{S}=1, n_{R}=1, n_{D}=L$ )

$$
\begin{aligned}
\mathbf{p}_{(1,1, L)}^{\text {outage }} & =\left[\left(1-e^{-z}\right)\left(1-e^{-z} \sum_{k=0}^{L-1} \frac{z^{k}}{k !}\right)\right. \\
& \left.+e^{-z}\left(1-e^{-z} \sum_{k=0}^{2 L-1} \frac{z^{k}}{k !}\right)\right] .
\end{aligned}
$$




\section{B. Array at the Relay}

When $n_{S}=1, n_{R}=L$, and, $n_{D}=1, \mathbf{H}_{s d}=h_{s d}$ is scalar (S-D is SISO), $\mathbf{H}_{s r}=\mathbf{h}_{s r}$ is a length L column vector ( $\mathrm{S}-\mathrm{R}$ is SIMO), and $\mathbf{H}_{r d}=\mathbf{h}_{r d}$ is a length L row vector (R-D is MISO). The information outage probability for the S-D link is the corresponding expression for a SISO link

$$
\operatorname{Pr}\left(C_{s d}<2 R\right)=1-e^{-z}
$$

The information outage probability for the S-R channel is the corresponding expression for a SIMO link

$$
\operatorname{Pr}\left(C_{s r}<2 R\right)=1-e^{-z} \sum_{k=0}^{L-1} \frac{z^{k}}{k !} .
$$

The information outage probability obtained when diversity combining of the MISO R-D and S-D SISO paths is $\operatorname{Pr}\left(C_{(s d, r d)}<2 R\right)$

$$
\begin{aligned}
& =\operatorname{Pr}\left[\log \left(1+\rho\left(\frac{\|\left.\mathbf{h}_{r d}\right|^{2}}{L}+\left|h_{s d}\right|^{2}\right)\right)<2 R\right] \\
& =\operatorname{Pr}[(\underbrace{\frac{\|\left.\mathbf{h}_{r d}\right|^{2}}{L}}_{X}+\underbrace{\left|h_{s d}\right|^{2}}_{Y})<\underbrace{\left.\frac{2^{2 R}-1}{\rho}\right]}_{z}] \\
& =\frac{L^{L}}{(L-1) !} \int_{0}^{z} x^{L-1} e^{-L x}\left[1-e^{x-z}\right] d x .
\end{aligned}
$$

The derivation of this expression is as follows. Here $X, Y$ are independent random variables. $X$ follows a $\chi^{2}$ distribution with $n_{1}=2 L$ DOF, while $Y$ is exponentially distributed with parameter $\lambda=1$ or equivalently $\chi^{2}$ with $2 \mathrm{DOF}$. While the CDF of $L X+Y$ is quite simple to find (it is $\chi^{2}$ with $2(L+1)$ DOF), the CDF of $X+Y$ is not straightforward. The CDF of $(X+Y)$ is found using

$$
\begin{aligned}
\operatorname{Pr}(X+Y \leq z) & =\int_{x=0}^{z} \int_{y=0}^{z-x} f_{x y}(x, y) d y d x \\
& =\int_{x=0}^{z} \int_{y=0}^{z-x} f_{x}(x) f_{y}(y) d y d x
\end{aligned}
$$

where the second line comes from the independence of $X$ and $Y$. Substituting the individual pdf's $f_{X}(x)=$ $\frac{L(L x)^{L-1} e^{-L x}}{(L-1) !} u(x)$ and $f_{Y}(y)=e^{-y} u(y)$, where $u(x)$ is the unit step function, yields

$\operatorname{Pr}(X+Y<z)=\int_{x=0}^{z} \int_{y=0}^{z-x} \frac{L(L x)^{L-1} e^{-L x}}{(L-1) !} e^{-y} d y d x$.

Integrating over y yields (19)

Substituting (17), (18), and (19) into (12), we arrive at the following expression for information outage probability for this case (i.e. $n_{S}=1, n_{R}=L, n_{D}=1$ )

$$
\begin{gathered}
\mathbf{p}_{(1, L, 1)}^{\text {outage }}=\left[\left(1-e^{-z}\right)\left(1-e^{-z} \sum_{k=0}^{L-1} \frac{z^{k}}{k !}\right)+\right. \\
\left.\left(e^{-z} \sum_{k=0}^{L-1} \frac{z^{k}}{k !}\right)\left(\frac{L^{L}}{(L-1) !} \int_{0}^{z} x^{L-1} e^{-L x}\left[1-e^{x-z}\right] d x\right)\right] .
\end{gathered}
$$

\section{Array at the Source}

Finally, consider the case that $\left(n_{S}=L, n_{R}=1, n_{D}=1\right)$. In this case, $\mathbf{H}_{s d}=\mathbf{h}_{s d}$ and $\mathbf{H}_{s r}=\mathbf{h}_{s r}$ are length $\mathrm{L}$ row vectors (S-D and S-R are MISO), and $\mathbf{H}_{r d}=h_{r d}$ is scalar (R-D is SISO). The information outage probability for the S-D channel is that of an MISO channel with $L$ transmit antennas

$$
\operatorname{Pr}\left(C_{s d}<2 R\right)=1-e^{-L z} \sum_{k=0}^{L-1} \frac{(L z)^{k}}{k !} .
$$

The information outage probability for the S-R channel is also that of an MISO channel with $L$ transmit antennas

$$
\operatorname{Pr}\left(C_{s r}<2 R\right)=1-e^{-L z} \sum_{k=0}^{L-1} \frac{(L z)^{k}}{k !} .
$$

The information outage probability obtained when diversity combining of the MISO S-D and SISO R-D paths is $\operatorname{Pr}\left(C_{(s d, r d)}<2 R\right)$

$$
\begin{aligned}
& =\operatorname{Pr}\left[\log \left(1+\rho\left(\frac{\left\|\mathbf{h}_{s d}\right\|^{2}}{L}+\left|h_{r d}\right|^{2}\right)\right)<2 R\right] \\
& =\operatorname{Pr}[(\underbrace{\frac{\|\left.\mathbf{h}_{s d}\right|^{2}}{L}}_{X}+\underbrace{\left|h_{r d}\right|^{2}}_{Y})<\underbrace{\frac{2^{2 R}-1}{\rho}}_{z}] \\
& =\frac{L^{L}}{(L-1) !} \int_{0}^{z} x^{L-1} e^{-L x}\left[1-e^{x-z}\right] d x .
\end{aligned}
$$

The derivation is identical to that for (19).

Substituting (21), (22), and (23) into (12), we arrive at the following expression for information outage probability for this case (i.e. $n_{S}=L, n_{R}=1, n_{D}=1$ )

$\mathbf{p}_{(L, 1,1)}^{\text {outage }}$

$$
\begin{aligned}
&= {\left[\left(1-e^{-L z} \sum_{k=0}^{L-1} \frac{(L z)^{k}}{k !}\right)^{2}+\left(e^{-L z} \sum_{k=0}^{L-1} \frac{(L z)^{k}}{k !}\right)\right.} \\
&\left.\left(\frac{L^{L}}{(L-1) !} \int_{0}^{z} x^{L-1} e^{-L x}\left[1-e^{x-z}\right] d x\right)\right] . \\
& \text { V. NUMERICAL RESULtS }
\end{aligned}
$$

In this section we present numerical results for the three terminal relay network with $R=1$ bit per channel use. While the information outage probability when the array is at the destination may be found by directly evaluating (16), the expressions for when the array is at the relay and at the source are expressed in terms of an integral. The two expressions, (20) and (24), may be evaluated using either a numerical integration or a Monte Carlo integration.

Fig. 2 presents the outage performance of adaptive DF when one of the three terminals has a two antenna array. For comparison purposes, we also show performance of a direct transmission (essentially $n_{R}=0$ ) with $n_{D}=2,3$ antennas at the destination. Note that all of the considered relaying schemes show diversity (as exhibited by the slope of the curves) that is greater than that of the direct transmission with $n_{D}=2$. When compared against direct transmission with $n_{D}=3$, the relaying schemes have a similar slope, but there 


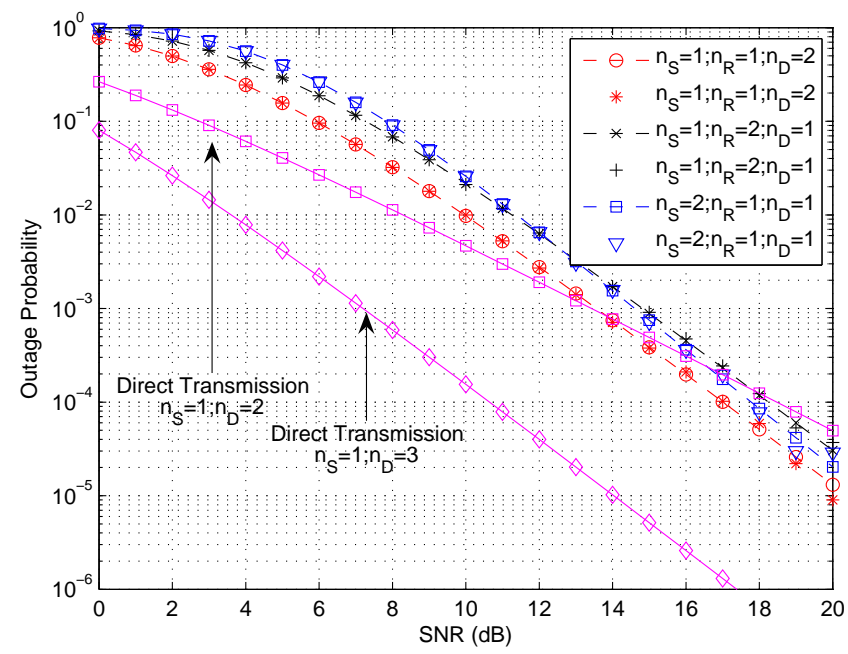

Fig. 2. Outage probability performance comparison between Monte Carlo integration (symbols) and numerical integration (dashed lines) for the configurations $\left(n_{S}=1, n_{R}=1, n_{D}=2\right),\left(n_{S}=1, n_{R}=2, n_{D}=1\right)$ and $\left(n_{S}=2, n_{R}=1, n_{D}=1\right)$ in quasi-static Rayleigh fading.

is a fixed SNR loss. The loss is due to several reasons. First, if the array is at the source or relay, then the power transmitted over each element must be cut in half. Also, there is a loss because there is a chance that the relay decodes incorrectly and is therefore unable to forward the message.

The analytical results also indicate that the most favorable choice for locating the two antennas is at the destination. This can be attributed to the receive diversity gain obtained by both the paths i.e. the S-D path and R-D path. There is SNR loss factor of $L$ in the received signal due to the lack of forward channel knowledge in the S-D, S-R paths or the R-D path in antenna configurations $\left(n_{S}=2, n_{R}=1, n_{D}=1\right)$ and $\left(n_{S}=1, n_{R}=2, n_{D}=1\right)$. Also, the relay retransmits the message only if it can decode correctly. The above reasons lead to the poor performance when multiple antennas are located at the source or relay. These outage probability results serve as the lower bound on the error rate of an arbitrary code word with the same fixed spectral efficiency.

Fig. 3 indicates the impact of increasing the number of receive antennas at the destination in a single relay cooperative network. The asymptotic slope of the curve increases with an increase in the number of antennas at the destination leading to a considerable performance gain. This performance gain can be further exploited by increasing the number of relay nodes. A performance tradeoffs similar to [5] for a multirelay network can be realized by increasing the number of antennas and the relays simultaneously. This analysis would lead to optimization of relay resources in wireless networks.

Figs. 4 and 5 show the information outage of the relay network with an increasing number of antennas located at the relay and source, respectively. In the low SNR regime, the performance when locating multiple antennas at the relay is better than when locating them at the source. In the

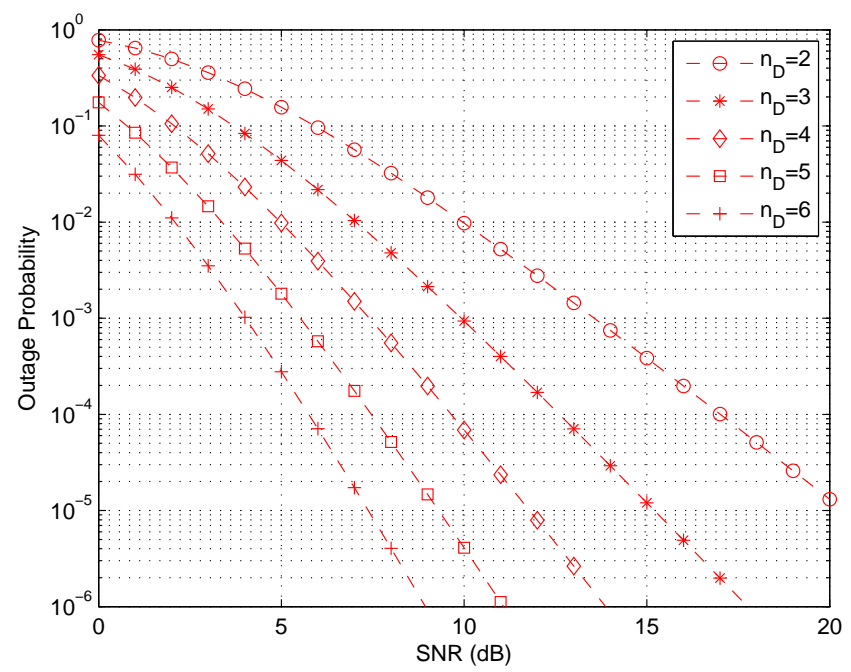

Fig. 3. Outage probability performance (numerical integration) with $n_{S}=$ $1 ; n_{R}=1$ and $n_{D}=2,3,4,5,6$ antennas in quasi-static Rayleigh fading.

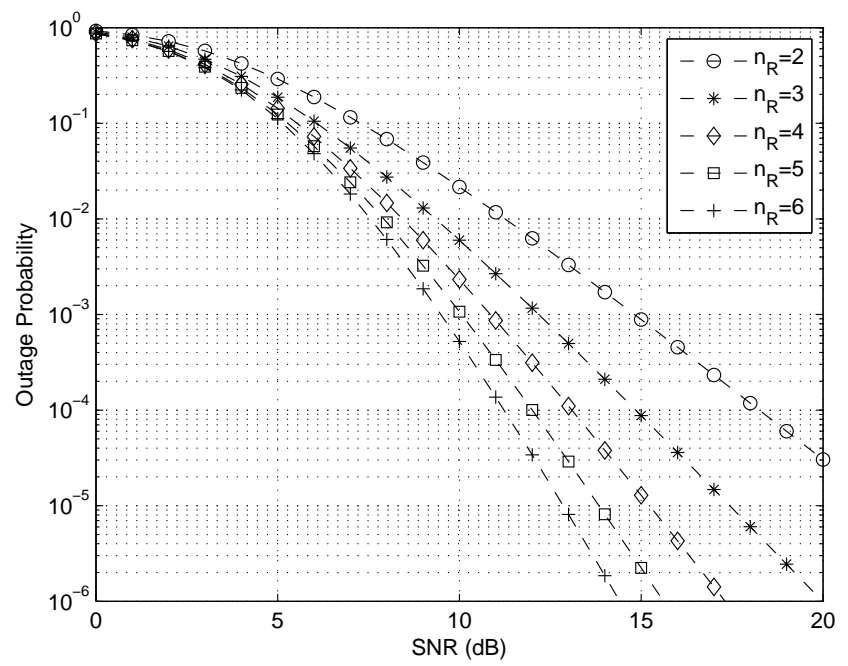

Fig. 4. Outage probability performance (numerical integration) with $n_{S}=$ $1 ; n_{R}=2,3,4,5,6$ and $n_{D}=1$ antennas in quasi-static Rayleigh fading.

configuration $\left(n_{S}=L, n_{R}=1, n_{D}=1\right)$ there is a loss in the received SNR at the relay due to the the lack of channel knowledge in the S-R path. Also, the relay retransmits only when the message is correct. The above reasons lead to poor performance when compared to $\left(n_{S}=1, n_{R}=L, n_{D}=1\right)$ in the low SNR regime.

The Fig. 6 overlays the results shown in Figs. 3 through 5 to provide a unified view of performance with an array of 2 to 6 elements located at one of the terminals. We observe that in SNR regime greater than $12 \mathrm{~dB}$, the outage performance obtained by increasing the number of antenna arrays at the relay and source terminals is relatively same. When we locate $L$ antennas at the source, a loss factor of $L$ is involved in the S-R path and the S-D path. Also, the relay forwards the 


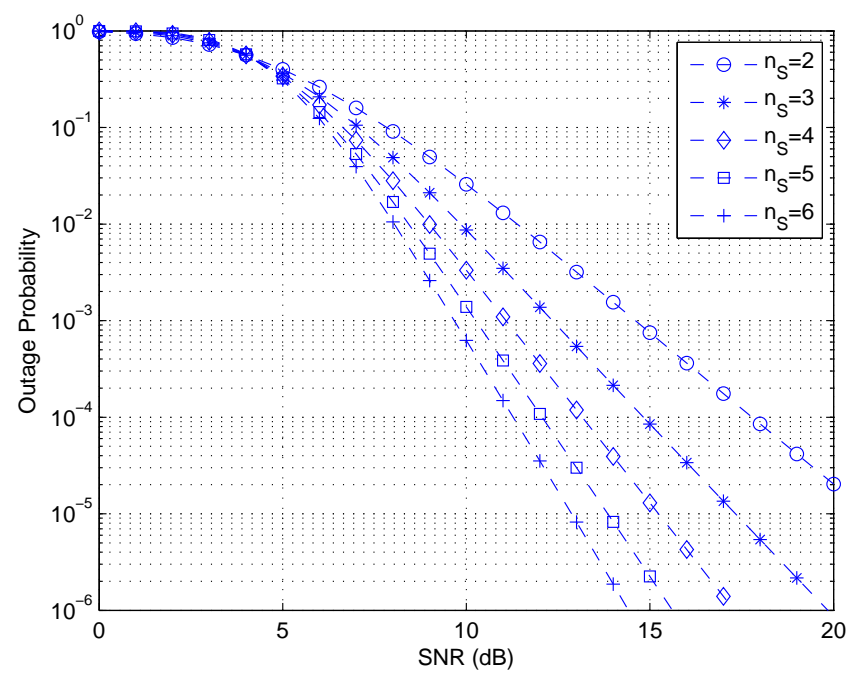

Fig. 5. Outage probability performance with $n_{S}=2,3,4,5,6 ; n_{R}=1$ and $n_{D}=1$ antennas in quasi-static Rayleigh fading.

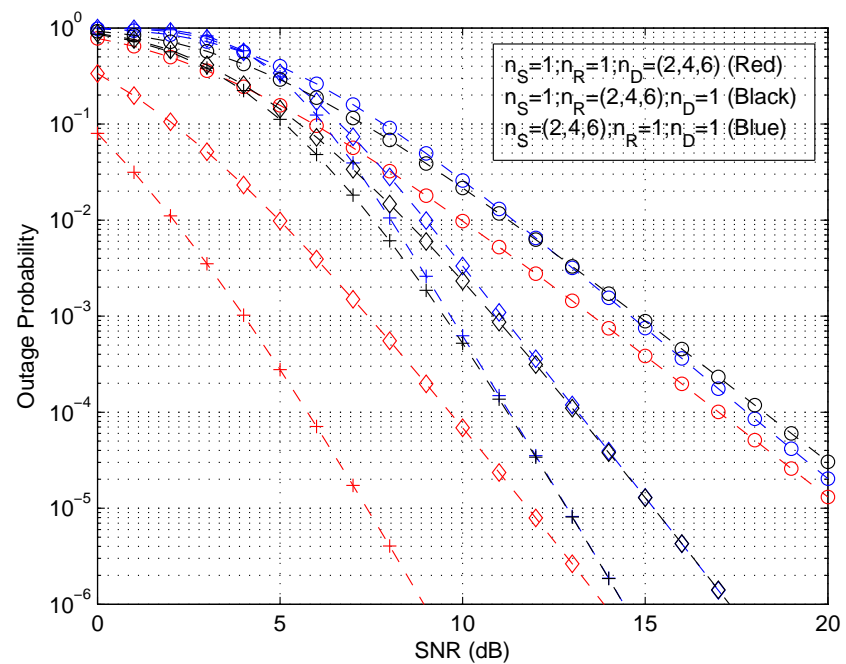

Fig. 6. Outage probability performance (numerical integration) with 2,4 , and 6 number of antennas at each node in quasi-static Rayleigh fading.

message only if it decodes correctly. In the case of locating multiple antennas at the relay the S-R path is a SIMO channel which accounts for the SNR gain when compared to locating $L$ antennas at the source. Also, the R-D path is a MISO channel, hence a loss factor $L$ is involved whenever the relay decodes the message correctly and forwards it to the destination. Hence in high SNR regime greater than $12 \mathrm{~dB}$ the performance of locating $L$ antennas at the source, relay is the same. It can also be seen that for the same outage and number of antennas, in each antenna configuration, the receive diversity has a considerable SNR gain of over $5 \mathrm{~dB}$. The SNR gain is due to the reason that there is no loss factor of $L$ involved in received SNR as the multiple antennas are located at the destination. Hence we conclude that incorporating multiple antennas at the destination yields the best results when there is a requirement to place all the available antennas at a single node.

\section{CONCLUSION}

In this paper, we have studied and analyzed the importance of an array within a three terminal relay network. An information outage analysis was presented for decode-andforward relaying with diversity combining at the destination. Our findings indicate that, given a set of additional antennas, the best choice is to locate all the additional antennas at the destination. The next best alternative is to position the additional antennas at the relay. The worst choice would be to put the additional antennas at the source. Future work could consider the following issues: (1) the impact of using code combining instead of diversity combining, (2) the use of other adaptive relaying schemes (such as hybrid-ARQ based relaying), (3) multi-relay networks and networks where more than one node has an array, (4) the influence of the choice of space-time code and modulation, and (5) the influence of topology on the problem.

\section{ACKNOWLEDGMENTS}

This work was supported by the Lane Endowment and by Augusta Systems in support of phase-II STTR contract number N68335-05-C-0303. The authors would like to thank Kanchan Vardhe for her technical assistance.

\section{REFERENCES}

[1] G. J. Foschini and M. J.Gans, "On limits of wireless communications in a fading environment when using multiple antennas," Wireless Personal Commun., vol. 6, pp. 311-355, March 1998.

[2] David Tse and Pramod Viswanath, Fundamentals of Wireless Communication. 1st ed., New York, NY: Cambridge University Press, 2005.

[3] A. Sendonaris, E. Erkip, and B. Aazhang, "User cooperation diversitypart I and part II," IEEE Trans. Commun., vol. 51, pp. 1927-1948, Nov. 2003.

[4] J. N. Laneman, D. N. C. Tse, and G. W. Wornell, "Cooperative diversity in wireless networks:efficient protocols and outage behaviour," IEEE Trans. Inform. Theory, vol. 50, pp. 3062-3080, Dec. 2004.

[5] J. N. Laneman and G. W. Wornell, "Distributed space-time coded protocols for exploiting cooperative diversity in wireless networks," IEEE Trans. Inform. Theory, vol. 49, pp. 2415-2525, Oct. 2003.

[6] T. Hunter, S. Sanayei, and A. Nosratinia, "Outage analysis of coded cooperation," IEEE Trans. Inform. Theory, vol. 52, pp. 375-391, Feb. 2006.

[7] M. Dohler, E. Lefranc, and H. Aghvami, "Space time block codes for virtual antenna arrays," in Proc. IEEE Personal Indoor and Mobile Radio Commun. Conf, (Lisbon, Portugal), Sep. 2002.

[8] P. Herhold, E. Zimmermann, and G. Fettweis, "On the performance of cooperative amplify-and-forward relay networks," in 5th International ITG Conference on Source and Channel Coding (SCC), (Erlangen, Germany), Jan. 2004.

[9] D. P. Palomar, A. Agustin, O. Muñoz, and J. Vidal, "Decode-and-forward protocol for cooperative diversity in multi-antenna wireless networks," in Proc. CISS, (Princeton, NJ), Mar. 2004.

[10] B. Zhao, Multiterminal Relay Networks: Performance Bounds, Protocol Design and Channel Coding Strategies. PhD thesis, West Virginia University, Morgantown, WV, April. 2004.

[11] J.G. Proakis, Digital Communications. 3rd ed., New York, NY: McGrawHill, 1995.

[12] J. N. Laneman, Cooperative diversity in wireless networks: Algorithms and architectures. $\mathrm{PhD}$ thesis, Massachusetts Institute of Technology, Cambridge, MA, August. 2002. 